\title{
Metabolically healthy but obese women: effect of an energy-restricted diet
}

\author{
A. D. Karelis • V. Messier • M. Brochu • \\ R. Rabasa-Lhoret
}

Received: 24 January 2008 / Accepted: 4 April 2008 / Published online: 27 May 2008

(C) Springer-Verlag 2008

Keywords Euglycaemic-hyperinsulinaemic clamp . Insulin resistance $\cdot$ Obesity Postmenopausal women

\author{
Abbreviations \\ FFM fat-free mass \\ FM fat mass \\ MHO metabolically healthy but obese
}

To the Editor: A unique subset of obese individuals has been identified that appears to be protected against obesityrelated metabolic disturbances $[1,2]$. These individuals, now known as 'metabolically healthy but obese' (MHO) individuals, display a favourable metabolic profile, characterised by high levels of insulin sensitivity, normal lipid

\footnotetext{
A. D. Karelis $(\bowtie)$

Department of Kinanthropology,

University of Quebec at Montreal,

Case postale 8888 , succursale Centre-ville,

Montreal, QC, Canada H3C 3P8

e-mail: karelis.antony@uqam.ca

V. Messier $\cdot$ R. Rabasa-Lhoret

Department of Nutrition,

University of Montreal,

Montreal, QC, Canada

M. Brochu

Faculty of Physical Activity and Sports, University of Sherbrooke,

Sherbrooke, QC, Canada

R. Rabasa-Lhoret

Montreal Diabetes Research Centre (MDRC),

Montreal University Hospital (CHUM),

Montreal, QC, Canada
}

and inflammation profiles and no sign of hypertension, despite having excessive body fatness. In fact, the metabolic profiles of MHO postmenopausal women are virtually indistinguishable from those of young lean women [3]. Interestingly, a recent longitudinal study reported that the protective metabolic profile observed in $\mathrm{MHO}$ individuals was associated with lower incidences of type 2 diabetes and cardiovascular diseases [4]. Moreover, evidence suggests that MHO individuals may account for as much as $20-30 \%$ of the obese population [5].

An important question that seems to be unresolved is whether MHO individuals would gain any metabolic benefit from weight loss. Indeed, several studies have shown that weight loss improves insulin sensitivity and metabolic abnormalities and reduces the risk for type 2 diabetes in obese individuals $[6,7]$. However, attempts to achieve weight loss in MHO individuals, by way of diet, may be actually counterproductive and potentially harmful. One may even question the need to aggressively treat $\mathrm{MHO}$ individuals given their favourable metabolic profile. Therefore, the aim of the present study was to investigate the effect of a 6 month energy-restricted diet on insulin sensitivity using the euglycaemic-hyperinsulinaemic clamp technique in a sample of MHO postmenopausal women.

This study was approved by the ethics committee of the University of Montreal. After reading and signing the consent form, each participant was invited to the Metabolic Unit for testing. The women then entered a medically supervised 6 month weight loss programme, which aimed to reduce body weight by $10 \%$. To achieve a level of energy restriction, the baseline resting metabolic rate was extrapolated over a $24 \mathrm{~h}$ period $(\mathrm{kcal} / \mathrm{min} \times 1,440 \mathrm{~min})$ and multiplied by an activity factor of 1.4 , which corresponds to a sedentary state. Thereafter, instructions on how to follow a hypoenergetic diet 
Table 1 Variables measured in the participants at baseline and at the end of the 6 month energy-restricted diet

\begin{tabular}{|c|c|c|c|c|}
\hline \multirow[t]{2}{*}{ Parameter } & \multicolumn{2}{|l|}{ Baseline } & \multicolumn{2}{|l|}{ Study end } \\
\hline & MHO & At-risk & MHO & At-risk \\
\hline Body weight (kg) & $89.6 \pm 11.8$ & $93.0 \pm 11.3$ & $84.6 \pm 11.2^{\dagger}$ & $86.5 \pm 12.2^{\dagger}$ \\
\hline $\mathrm{FM}(\mathrm{kg})$ & $42.8 \pm 7.1$ & $42.2 \pm 6.2$ & $39.8 \pm 7.8^{\dagger}$ & $37.8 \pm 8.7^{\dagger}$ \\
\hline FFM (kg) & $45.7 \pm 5.1$ & $50.6 \pm 7.3^{*}$ & $44.5 \pm 4.3^{\dagger}$ & $48.6 \pm 6.3^{*, \dagger}$ \\
\hline Insulin sensitivity $\left(\mu \mathrm{mol} \min ^{-1}[\mathrm{~kg} \mathrm{FFM}]^{-1}\right)$ & $86.7 \pm 13.9$ & $42.0 \pm 7.6^{* *}$ & $75.6 \pm 18.0^{\dagger}$ & $53.0 \pm 12.1^{* *, \dagger}$ \\
\hline Insulin sensitivity ${ }^{\mathrm{a}}\left(\mu \mathrm{mol} \mathrm{min}{ }^{-1}[\mathrm{~kg} \mathrm{FFM}]^{-1}[\mathrm{pmol} / 1]^{-1}\right)$ & $70.0 \pm 24.1$ & $30.0 \pm 8.0^{* *}$ & $61.2 \pm 22.8^{\dagger}$ & $37.7 \pm 11.5^{* *, \dagger}$ \\
\hline
\end{tabular}

${ }^{\text {a }}$ Normalised for the achieved insulin concentration plateau

${ }^{*} p<0.05,{ }^{* *} p<0.01$ vs MHO at same time point; ${ }^{\dagger} p<0.01$ vs baseline

$(2,000-3,500 \mathrm{~kJ} /$ day [500-800 kcal/day] deficit) were given by a registered dietician on a one-to-one basis. The patients were thus prescribed a balanced diet that provided 4,600$7,550 \mathrm{~kJ} /$ day $(1,100-1,800 \mathrm{kcal} /$ day $)$. Food was self-selected and the macronutrient composition was standardised $(55 \%$, $30 \%$ and $15 \%$ of total energy intake from carbohydrates, fat and protein, respectively) under the supervision of a registered dietician. In addition, participants met bi-monthly with the registered dietician for nutrition classes (1.0-1.5 h) to discuss healthy eating practices. All participants were instructed not to change physical activity habits during the weight loss protocol.

Before and after the 6 month diet, body weight was stabilised for 4 weeks to within $\pm 1 \mathrm{~kg}$ by monitoring body weight for each participant on a weekly basis at our laboratory. The euglycaemic-hyperinsulinaemic clamp was conducted using an insulin infusion rate of $75 \mathrm{mU}$ $\mathrm{m}^{-2} \min ^{-1}$ for $180 \mathrm{~min}$. Plasma glucose was measured every 10 min with a glucose analyser (Beckman Instruments, Fullerton, CA, USA) and maintained at fasting level by infusing $20 \%$ (wt/vol.) dextrose at a variable rate. Glucose disposal was calculated as the mean rate of glucose infusion measured during the last $30 \mathrm{~min}$ of the clamp (steady state) and was expressed as $\mu \mathrm{mol}$ per min per $\mathrm{kg}$ of fat free mass (FFM). Fat mass (FM) and FFM were measured by dualenergy X-ray absorptiometry with a Lunar Prodigy System (version 6.10.019; General Electric Lunar, Madison, WI, USA). MHO and 'at-risk' individuals were identified as previously described [2], using the euglycaemic-hyperinsulinaemic clamp technique. Briefly, MHO and at-risk individuals were identified by dividing the entire cohort into quartiles based on insulin sensitivity values ( $M$ value/FFM; $n=121$ ). Women with values for $M$ value/FFM in the upper quartile $\left(\geq 73.9 \mu \mathrm{mol} \mathrm{min}{ }^{-1}[\mathrm{~kg} \mathrm{FFM}]^{-1}\right)$ were classified as having high insulin sensitivity and placed in the MHO group $(n=30)$, whereas women with $M$ value/FFM in the lower quartile $\left(\leq 49.9 \mu \mathrm{mol} \mathrm{min}^{-1}[\mathrm{~kg} \mathrm{FFM}]^{-1}\right)$ were classified as having low insulin sensitivity and categorised as at-risk participants $(n=30)$. It should be noted that ten MHO and six at-risk participants did not complete the study. Therefore, analyses were performed using the results for the $20 \mathrm{MHO}$ and 24 at-risk participants that remained at the end of the intervention. Data are expressed as the mean $\pm \mathrm{SD}$. A nonpaired Student's $t$ test was used for the comparison between groups at baseline and after 6 months. A repeated measures ANOVA was used to detect changes with time within the treatment condition (pretreatment vs post-treatment) and between groups (MHO vs at-risk). A $p$ value of $<0.05$ was considered significant.

Both groups were similar in terms of age (MHO: $57.7 \pm$ 4.5 vs at-risk: $58.3 \pm 4.7$ years). No significant differences between groups were observed for body weight or FM before or after the diet (Table 1). As for FFM, significant differences $(p<0.05)$ between groups were noted before and after the diet. By design, MHO individuals had significantly higher $(p<0.01)$ insulin sensitivity than atrisk participants at baseline, and this difference persisted at the end of the 6 month diet (Fig. 1). Furthermore, by the end of the diet, insulin sensitivity levels had significantly increased $(p<0.01)$ by $26.1 \%$ in at-risk participants and had significantly decreased $(p<0.01)$ by $12.8 \%$ in MHO individuals (Fig. 1). We also analysed insulin sensitivity normalised for the achieved plasma insulin concentration

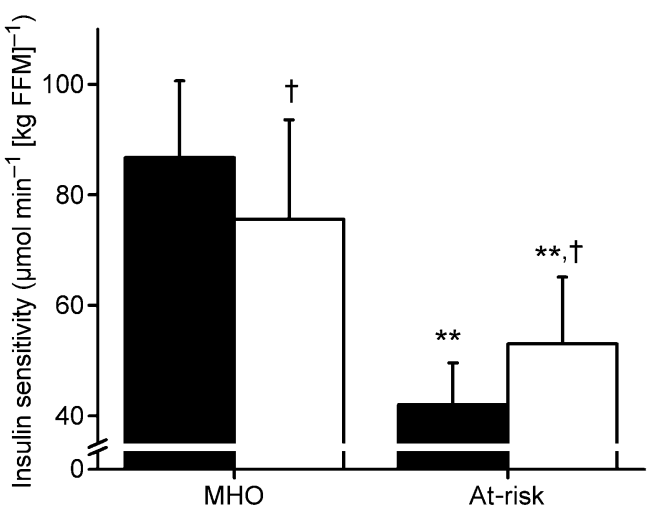

Fig. 1 Changes in insulin sensitivity levels before and after the energy-restricted diet in $\mathrm{MHO}$ and at-risk postmenopausal women. Values are mean \pm SD. ${ }^{* *} p<0.01$ vs at-risk individuals at same time point; ${ }^{\dagger} p<0.01$ vs baseline 
plateau, before and after the diet. Results showed that, at study end, insulin sensitivity had significantly increased $(p<0.01)$ by $\sim 26 \%$ in at-risk participants, whereas it had decreased $(p<0.01)$ by $\sim 13 \%$ in MHO individuals. The improvement in insulin sensitivity levels in at-risk participants after the diet was expected. However, the deterioration of insulin sensitivity levels in MHO participants was surprising and requires further investigation of the possible mechanism(s) involved. It should be noted that the MHO and at-risk groups exhibited similar significant reductions in body weight (6\% vs $7 \%$, respectively; $p<0.01)$ and FM loss ( $7 \%$ vs $10 \%$, respectively; $p<0.01)$ after the diet. Interestingly, we examined indirect calorimetry data (data not shown) and observed no significant changes in the respiratory quotient in either group between baseline and after the diet. This could suggest that, in contrast to the increase in insulin sensitivity associated with exercise, the improvement in insulin sensitivity in atrisk participants was not associated with changes in fasting oxidative substrate disposal.

Our results suggest that $\mathrm{MHO}$ individuals may respond differently to an energy-restricted diet compared with at-risk individuals who achieve a similar weight loss, in that insulin sensitivity significantly improved in at-risk participants, but significantly deteriorated in $\mathrm{MHO}$ individuals in response to the 6 month diet. It should be noted that our findings are limited to a small population of sedentary obese postmenopausal women without type 2 diabetes. A better understanding of MHO individuals has important implications for medical education and research. It is important to educate healthcare professionals and physicians regarding the different needs of subsets of obese individuals. The tendency to treat obese individuals with a 'one size fits all' approach may be counterproductive in the MHO individual if the goal is to improve insulin sensitivity. Indeed, the identification of the MHO individual in a clinical setting could have important implications for therapeutic medical decisionmaking such as whether or not to treat obese individuals with a diet. Our results should be considered preliminary, but they may hopefully stimulate interest in the need for greater participant characterisation in research protocols.

Acknowledgements This study was supported by grants from the Canadian Institute of Health Research New Emerging Teams in Obesity (University of Montreal and University of Ottawa; MONET project). R. Rabasa-Lhoret is supported by the Quebec Health Research Funds (Fonds de la Recherche en Santé du Québec).

Duality of interest The authors declare that there is no duality of interest associated with this manuscript.

\section{References}

1. Brochu M, Tchernof A, Dionne IJ et al (2001) What are the physical characteristics associated with a normal metabolic profile despite a high level of obesity in postmenopausal women? J Clin Endocrinol Metab 86:1020-1025

2. Karelis AD, Faraj M, Bastard JP et al (2005) The metabolically healthy but obese individual presents a favorable inflammation profile. J Clin Endocrinol Metab 90:4145-4150

3. Dvorak RV, DeNino WF, Ades PA, Poehlman ET (1999) Phenotypic characteristics associated with insulin resistance in metabolically obese but normal-weight young women. Diabetes 48:2210-2214

4. Meigs JB, Wilson PW, Fox CS et al (2006) Body mass index, metabolic syndrome, and risk of type 2 diabetes or cardiovascular disease. J Clin Endocrinol Metab 91:2906-2912

5. Karelis AD, St-Pierre DH, Conus F, Rabasa-Lhoret R, Poehlman ET (2004) Metabolic and body composition factors in subgroups of obesity: what do we know? J Clin Endocrinol Metab 89:25692575

6. McAuley KA, Williams SM, Mann JI et al (2002) Intensive lifestyle changes are necessary to improve insulin sensitivity: a randomized controlled trial. Diabetes Care 25:445-452

7. Muscelli E, Camastra S, Catalano C et al (1997) Metabolic and cardiovascular assessment in moderate obesity: effect of weight loss. J Clin Endocrinol Metab 82:2937-2943 